\title{
FREQUENCY SPECTRUM FOR INTEGRATION OF UNMANNED AIRCRAFT
}

\author{
Robert J. Kerczewski and Jeffrey D. Wilson, NASA Glenn Research Center, Cleveland, Ohio \\ William D. Bishop, Verizon, Cleveland, Ohio
}

\begin{abstract}
The goal of enabling the integration of unmanned aircraft systems (UAS) into the National Airspace System (NAS) in terms of UAS achieving routine access to the NAS has been established as a national goal in the United States. Among a number of technical barriers that must be overcome to meet this goal is the absence of standard, certifiable communications links supplying the control and nonpayload communications (CNPC) function, essentially providing the link over which a pilot on the ground can control the unmanned aircraft (UA). The International Civil Aviation Organization (ICAO) has determined that the CNPC link must operate over protected aviation spectrum. Therefore protected aviation spectrum must be allocated for this function, approved through the processes of the International Telecommunications Union Radiocommunication Sector (ITU-R).

Work has progressed in the definition of spectrum requirements for CNPC, and a portion of these requirements has been satisfied through new allocations approved at the ITU-R 2012 World Radiocommunication Conference (WRC-12). Additional work is ongoing or planned to satisfy the remaining spectrum requirements and define the specifications for the usage of CNPC spectrum allocations and develop supporting standards.
\end{abstract}

This paper provides an overview of the status of RF spectrum for UAS CNPC. Issues that have been identified and ongoing analysis and research that will be necessary to fulfill spectrum requirements for UAS CNPC will be discussed. The results of this work will provide for the safe integration of UA into the NAS in both the LOS (Line of Sight) and BLOS (Beyond Line of Sight) realms.

\section{Introduction}

There is an increasing need to fly unmanned aircraft (UA) in the National Airspace System (NAS) to perform missions of vital importance to National
Security and Defense, Emergency Management, and Science as well as for commercial applications (e.g. cargo transport). However, there are a number of critical technical barriers to the integration of unmanned aircraft systems (UAS) into the NAS. Several of these technical barriers are being addressed under NASA's UAS Integration in the NAS (UAS in the NAS) Project, including:

Separation Assurance/Sense and Avoid addressing the uncertainty that exists surrounding the ability to interoperate in Air Traffic Control (ATC) environments and maintain safe separation from other aircraft in the absence of an on-board pilot.

Human Systems Integration - addressing the lack of standards and guidelines with respect to UAS display/information as well as lack of Ground Control Station (GCS) design requirements to operate in the NAS.

Certification - addressing the lack of airworthiness requirements and safety-related data specific to the full range of UAS, or for their avionics systems or other components

Communications - addressing the lack of standard, certifiable data links and aviation safety spectrum to operate such links for civil UAS control communications.

The Communications Sub Project of the UAS in the NAS Project is focused on the UA control and non-payload communications (CNPC) link connecting the "pilot" on the ground with the UA in flight. This link supports safety critical functions. Loss or compromise of the CNPC link has potentially catastrophic consequences. Therefore, the International Civil Aviation Organization (ICAO) has determined that the CNPC links must operate over protected aviation spectrum, under International Telecommunications Union (ITU) designations Aeronautical Mobile (Route) Service (AM(R)S) and Aeronautical Mobile Satellite (Route) Service $(\operatorname{AMS}(\mathrm{R}) \mathrm{S})$. These are the two designations required to support "safety and regularity of flight". AM(R)S spectrum will support LOS terrestrial-based CNPC 
links while AMS(R)S spectrum will support BLOS satellite-based CNPC links.

Both LOS and BLOS spectrum requirements are being addressed through the ITU-R regulatory processes, beginning with the establishment by WRC-07 of an agenda item for UAS spectrum for WRC-12, followed by WRC-12 addressing that agenda item and establishing a new agenda item for WRC-15. If the decisions of WRC-15 result in all UAS spectrum requirements being met satisfactorily, the remaining work will entail development of standards for the use of the spectrum on both the national and international level, and technology development and evaluation efforts to provide technical support for the standards development process.

The following sections of this paper present information on the status of spectrum for CNPC and what has been accomplished so far; current work efforts addressing CNPC requirements, particularly for BLOS; and new work that will be required to completely resolve the spectrum requirement problem for UAS CNPC to enable the safe integration of UA into the NAS.

\section{Spectrum Requirements for CNPC}

The spectrum requirements for UAS CNPC have been defined by the ITU-R for the purpose of determining spectrum allocations. Since the application of UA to non-military purposes is in its infancy, projections of the expected UA density for the year 2030 were developed. From [1], two methodologies were employed to develop UA density estimates. Methodology 1 used studies that estimate the peak instantaneous aircraft count and assumes that $10 \%$ of these estimations would be UA, while methodology 2 projected the UA density based on estimated UAS usage rates.

The data transfer requirements for a single UA support several functions: command and control; air traffic control (ATC) relay; sense and avoid (S\&A); and video and weather radar. Command and control includes command and navigational signals sent to the UA from the UA control station (UACS) and position, telemetry and navigational information sent from the UA to the UACS. Air traffic control relay consists of voice and data commands from ATC to the UACS through UA. At some point in the future connectivity between ATC and the UACS may occur primarily, if not completely, through ground-based communication, bypassing the need for a relay through the UA. However such a capability does not currently exist. Sense and avoid capability allows the $\mathrm{UA}$ to maintain required separation distances between itself and other air traffic. The S\&A data are sent from the UA to the UACS to enable assessment of the correct operation of the S\&A function. In some operations and some phases of flight video will be sent from the UA to the UACS to assist in the S\&A function. Weather radar data may also be sent from the UA to the UACS to enable weather avoidance. The data requirements for these functions were considered for six phases of flight: taxiing; takeoff; initial climb; cruise (en-route); approach; and landing.

The data requirements thus derived were aggregated over the projected 2030 UA densities to arrive at spectrum requirements. For both UA density methodologies, the terrestrial LOS spectrum requirement was determined to be $34 \mathrm{MHz}$. For the satellite BLOS component, the spectrum requirement is between $46 \mathrm{MHz}$ and $56 \mathrm{MHz}$, dependent upon the type of satellite system used (spot beam or regional beam). Thus the maximum spectrum requirements for UAS are:

- $\quad 34 \mathrm{MHz}$ for LOS terrestrial systems,

- $\quad 56 \mathrm{MHz}$ for BLOS satellite systems.

\section{Line of Sight CNPC Spectrum Allocations and WRC-12}

Spectrum allocations for LOS CNPC using the $\mathrm{AM}(\mathrm{R}) \mathrm{S}$ were considered in [2]. This report considered use of the existing $\mathrm{AM}(\mathrm{R}) \mathrm{S}$ allocation in the band 960-1164 MHz. The report's conclusion was that due to the navigational uses, in particular for Distance Measuring Equipment (DME) and Tactical Ari Navigation systems (TACAN), that occupy most of this band, it cannot be used to meet the entire 34 $\mathrm{MHz}$ terrestrial spectrum requirement for UAS operations. Figure 1 from [2] shows the spectral occupancy from DME and TACAN assignments in the United States and Canada.

Note that below $977 \mathrm{MHz}$ DME and TACAN are not used. This band of $960-977 \mathrm{MHz}$ is not 


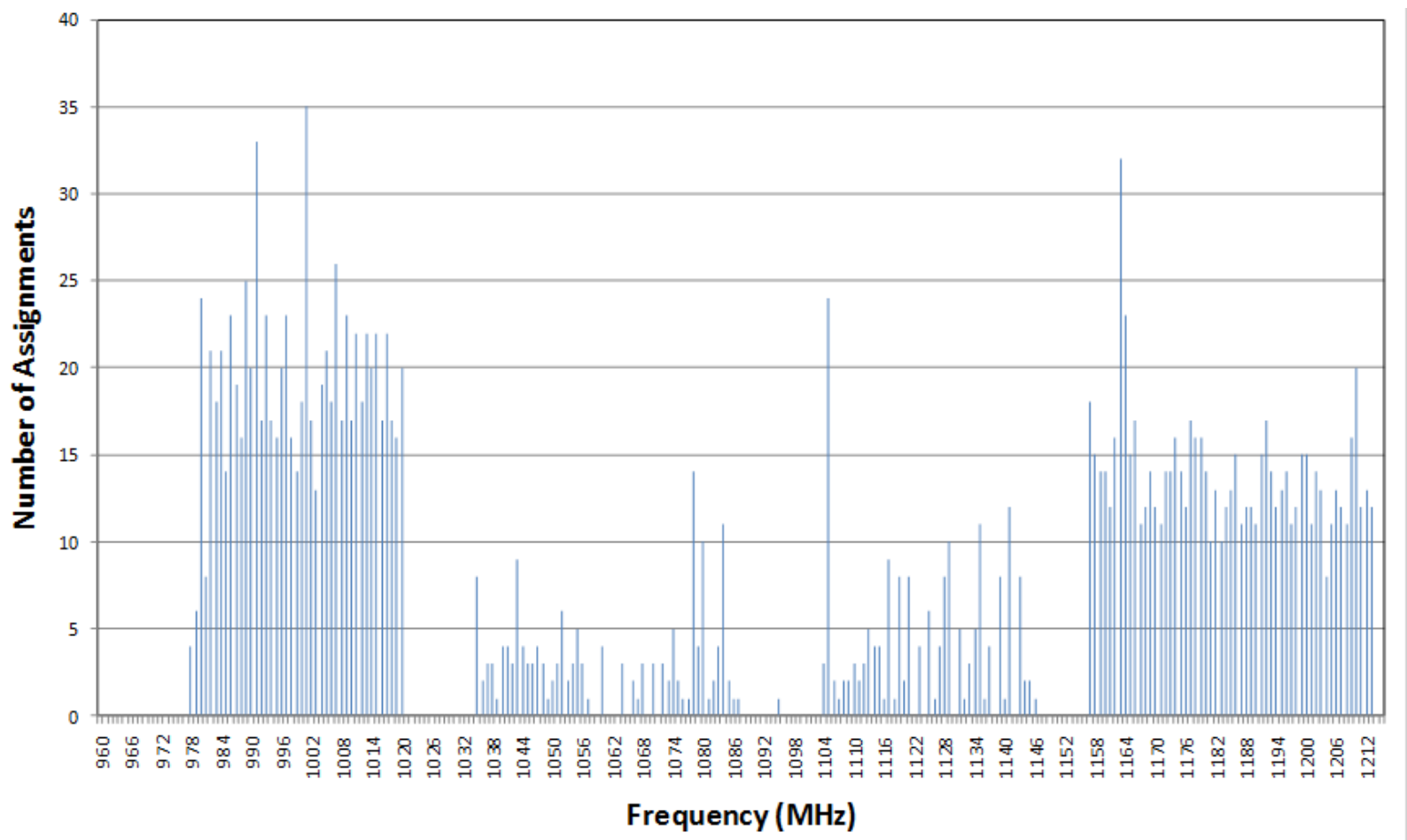

Figure 1. Spectral Occupancy of DME and TACAN ground assignments (from [2])

governed internationally, and its usage is controlled on a nation-by-nation basis. It is sometimes used by shipborne TACAN and by DME on a national basis or land-based TACAN, and is considered suitable for UAS CNPC, at least in areas where TACAN is not in use. Thus 960-977 MHz can supply approximately $17 \mathrm{MHz}$ of the $34 \mathrm{MHz}$ required for LOS terrestrial CNPC, depending on guard band requirements needed to protect systems in adjacent bands, such as Universal Access Transceiver (UAT) located at 978 MHz.

At the World Radiocommunication Conference 2012 (WRC-12), Agenda Item 1.3 (AI 1.3) (Resolution 421 of WRC-07) included the study of spectrum requirements for UAS and required regulatory actions including additional allocations. The reports of $[1,2]$ were responsive to this agenda item.

Although the 960-1164 MHz band had already been allocated to $\mathrm{AM}(\mathrm{R}) \mathrm{S}$, and has preferred propagation properties for aeronautical communications compared to higher frequency bands, as was reported in [2] the available usable spectrum for LOS terrestrial CNPC is insufficient.
The next candidate band for consideration then became 5030-5091 MHz. This band has an aeronautical radionavigation service (ARNS) allocation intended to support Microwave Landing System (MLS) installations. However, MLS has not been deployed as originally envisioned, with only a few installations worldwide outside of China, making it a viable alternative for other service allocations.

To address WRC-12 AI 1.3, line-of-sight CNPC link characteristics were established for the 5000$5150 \mathrm{MHz}$ band [3]. These characteristics enabled a study of the compatibility between UAS LOS CNPC and ARNS, and between UAS LOS CNPC and AMS(R)S [4]. The study concluded that sharing between $A M(R) S$ with both ARNS and AMS(R)S is possible, provided that sufficient mitigation techniques were employed. The mitigation techniques studies included frequency planning, geographical separation and power control. However, the report noted that compatible operation of UAS in the same occupied bandwidth $(150 \mathrm{kHz})$ with an MLS installation would be difficult to achieve. 
On the basis of these studies, WRC-12 approved an allocation to the $A M(R) S$ in the band 5030-5091 $\mathrm{MHz}$ [5]. So this band now contains ARNS (MLS mostly in China); $\operatorname{AMS}(\mathrm{R}) \mathrm{S}$ (no satellites currently provide service in this band); and $\operatorname{AM}(\mathrm{R}) \mathrm{S}$ (a new allocation). The allocation 5.C103 notes: "The use of the frequency band $5030-5091 \mathrm{MHz}$ by the aeronautical mobile (R) service is limited to internationally standardized aeronautical systems." All three services in the 5030-5091 MHz band carry the same limitation. This in essence means that it is up to the international aeronautical community, namely ICAO, to develop the standards governing the use of this band for the aeronautical services.

To meet the requirement of $34 \mathrm{MHz}$ of spectrum to support LOS CNPC, the $17 \mathrm{MHz}$ in the band 960$977 \mathrm{MHz}$ and a portion of the $61 \mathrm{MHz}$ now available in the 5030-5091 MHz band can be used. Although $61 \mathrm{MHz}$ in the $5030-5091 \mathrm{MHz}$ band would easily meet the $34 \mathrm{MHz}$ requirement, not all of that bandwidth is necessarily available due to the existence of MLS installations in some places and the AMS(R)S allocation that may also be required to meet the BLOS CNPC requirement. In addition, it is desirable to have two separate frequency bands available in order to meet stringent CNPC reliability requirements that will exist under some circumstances by using two redundant radio systems in different bands.

\section{Beyond Line of Sight CNPC Spectrum Allocations and WRC-15}

At the conclusion of WRC-12, the $34 \mathrm{MHz}$ spectrum requirement for LOS terrestrial CNPC can now be met, using approximately $17 \mathrm{MHz}$ within 960-977 $\mathrm{MHz}$ and a portion of the new $\mathrm{AM}(\mathrm{R}) \mathrm{S}$ allocation in 5030-5091 MHz.

Since $56 \mathrm{MHz}$ is required to support BLOS satellite CNPC, and at least $17 \mathrm{MHz}$ of the $5030-5091 \mathrm{MHz}$ band is needed for LOS CNPC, the remaining bandwidth is insufficient, less than $44 \mathrm{MHz}$ due to the need to protect services in adjacent bands, especially $5000-5030 \mathrm{MHz}$. A further complication is that there are no satellites in operation, nor are any planned, to offer services for UAS CNPC in that band. Although there is also an $\operatorname{AMS}(\mathrm{R}) \mathrm{S}$ allocation in L-Band (1545-1555 MHz space-to-earth and 1646.5-1656.5 MHz earth-to-space) where satellites are providing aeronautical services, it is too heavily used to be able to support UAS CNPC. So to meet the goal of enabling integration of UAS into nonsegregated airspace within the next several years, another approach is required.

The alternative that has been proposed is to use existing satellites in the Fixed Satellite Service (FSS) to provide BLOS CNPC. Although some UASs have used FSS for their CNPC links, this has occurred only by exception through the Certificate of Waiver or Authorization (COA) process, which occurs once per UAS operation and does not allow routine access of UAS. However this past use of FSS demonstrates the feasibility of this approach.

As mentioned above, ICAO has determined that the CNPC link must operate over protected aviation spectrum, which the FSS allocation does not meet. Therefore, special considerations must be met to allow CNPC operations over FSS satellites.

To study this and make decisions on the possible use of FSS for UAS CNPC, WRC-12 adopted Resolution 153: "To consider the use of frequency bands allocated to the fixed-satellite service not subject to Appendices 30, 30A and 30B for the control and nonpayload communications of unmanned aircraft systems in non-segregated airspaces" [5]. This resolution invites WRC-15 to consider, based on the results of the ITU-R studies, possible regulatory actions to support the use of FSS frequency bands for the UAS CNPC links. It also invites ITU-R, ICAO and other administrations and organizations to conduct the necessary studies leading to technical, regulatory and operational recommendations to WRC-15 to enable it to decide on the usage of FSS for the CNPC links for the operation of UAS.

The study process has evolved into two areas: technical studies on the compatibility of UA earth stations with other services in the FSS bands, and approaches to resolving regulatory issues that involve use of a non-safety service to provide aviation safety communications services. The technical studies are described in the next section. A description of the regulatory issues is presented below.

It is expected that WRC-15 can approve the use of FSS for UAS CNPC if compatibility between services can be shown, and if it can also be shown that the FSS can provide the level of service required for safe operation of the UAS. ICAO is responsible 
for establishing the communications performance requirements for safe UAS operation, and is in the process of doing so through the ICAO UAS Study Group. This work is not yet complete so in order to advance WRC-15 AI 1.5, ICAO has established and submitted to ITU-R the following conditions for use of satellite CNPC links [6]:

1) that the technical and regulatory actions should be limited to the case of UAS using satellites, as studied, and not set a precedent that puts other aeronautical safety services at risk;

2) that all frequency bands which carry aeronautical safety communications need to be clearly identified in the Radio Regulations;

3) that the assignments and use of the relevant frequency bands have to be consistent with Article 4.10 of the Radio Regulations which recognizes that safety services require special measures to ensure their freedom from harmful interference;

4) that knowledge of any assignment operating in those frequency bands has been successfully coordinated under Article 9 of the Radio Regulations (e.g. any caveats placed on that assignment have been addressed and resolved);

5) that all assignments used by satellite systems for the provision of UAS CNPC links are registered with favorable findings in the master international frequency register;

6) that interference to systems is reported in a transparent manner and addressed in the appropriate timescale;

7) that realistic worst case conditions with the inclusion of a safety margin can be applied during compatibility studies;

8) that any operational considerations for UAS will be handled in ICAO and not in the ITU.

Contributions to WRC-15 are being developed to show how these conditions would be met. Most importantly, operational characteristics would be defined in ICAO Standards and Recommended Practices (SARPS). Service agreements between a UAS operator and a satellite service provider would need to be certified as meeting the SARPS requirements. This includes the need for coordination agreements between satellite operators to cover such items as frequency planning between systems.
Ultimately only FSS systems that can meet the performance requirements specified in the SARPS will be able to be certified for use in all airspace.

\section{Analyses for WRC-15 Agenda item 1.5}

Technical studies on the compatibility of UAS earth stations with other existing services in the FSS bands are focused on the frequency sharing between the satellite communications terminal serving the UA, known as the UA earth station (UAES) and cofrequency terrestrial services, which are point-topoint microwave communications under the Fixed Service (FS) allocation. Since the UAES is assumed to be compliant with earth station requirements for FSS, and the UAES antenna is also assumed to be capable of maintaining accurate pointing toward the satellite, there are no further studies of the UAES satellite link required for compatibility analysis.

The BLOS satellite CNPC links that are being studied for sharing compatibility between the UAES and the terrestrial FS stations are shown in Figure 2. Two directions of interference are being studied: interference from the FS into the UAES (Link 2s FS station transmission interfering with Link 2 downlink from the satellite to the UAES in Figure 2), and interference from the UAES into the FS station (link 3 uplink transmission from the UAES to the satellite creating the interference Link $3 \mathrm{~s}$ into the FS station receiver). The frequency ranges being studied cover FSS allocations in the $\mathrm{Ku}$ and $\mathrm{Ka}$ Bands. Table 1 identifies the bands, ranges and associated links involved in the analysis.

Table 1. Frequency Ranges and Links for WRC15 Agenda Item 1.5 Compatibility Study

\begin{tabular}{|c|c|c|}
\hline $\begin{array}{c}\text { Frequency } \\
\text { Band }\end{array}$ & $\begin{array}{c}\text { Frequency } \\
\text { Range }\end{array}$ & $\begin{array}{c}\text { Link } \\
\text { Studied } \\
\text { (Figure 2) }\end{array}$ \\
\hline $\mathrm{Ku}$ & $\begin{array}{c}10.95-12.75 \\
\mathrm{GHz}\end{array}$ & 2 and 2s \\
\hline $\mathrm{Ka}$ & $\begin{array}{c}17.3-20.2 \\
\mathrm{GHz}\end{array}$ & 2 and 2s \\
\hline $\mathrm{Ku}$ & $\begin{array}{c}14.0-14.5 \\
\mathrm{GHz}\end{array}$ & 3 and 3s \\
\hline $\mathrm{Ka}$ & $27.5-30.0$ & 3and 3s \\
& $\mathrm{GHz}$ & \\
\hline
\end{tabular}




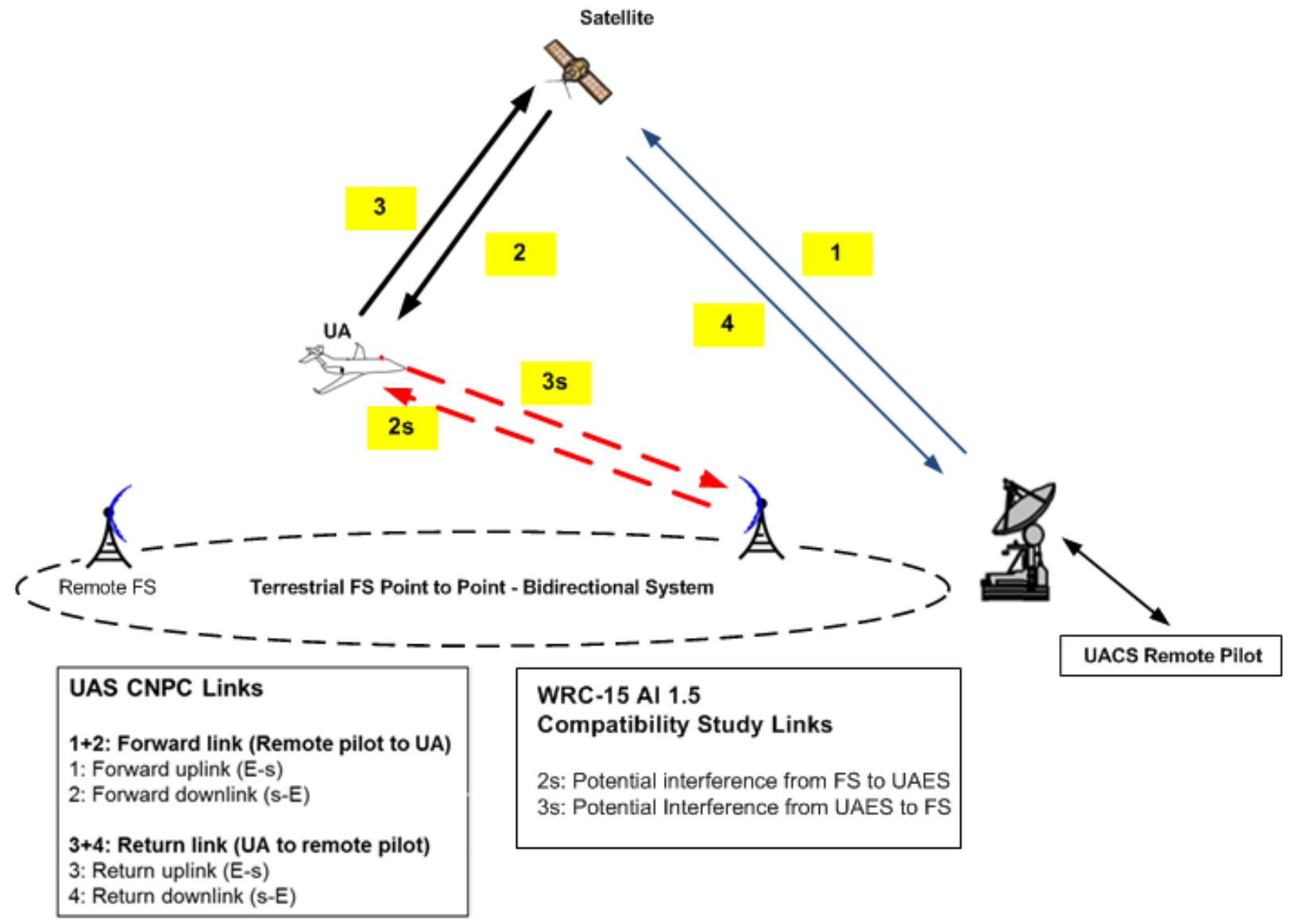

\section{Figure 2. Satellite and Terrestrial Communications Links Involved in UAS Compatibility Studies for WRC-15 Agenda Item 1.5}

The analysis of interference of FS station transmissions into the UAES receiver is being performed for two downlink (space-to-earth) FSS bands (Table 1). In the $10.95-12.75 \mathrm{GHz}$ band there are primary allocations to the FS covering the entire band. In the 17.3-20.2 GHz band there are primary allocations to the FS covering 18.1-20.2 GHz. It should be noted that there are allocations to other terrestrial services in these bands but no technical characteristics for these other services have been documented, thus no sharing studies can be completed for these services.

The United States' analyses of interference in these two bands, being carried out by spectrum experts from the Federal Aviation Administration, consider the interference effects from FS station transmitters in these bands. The FS transmission parameters used are those defined in [7]. Since there are potentially many FS stations that could be within the line of sight of a UAES, the analysis must include the aggregate effect of all of the stations. An example of a random distribution of FS stations based on known densities of FS stations is used as shown in Figure 3 [8]. For these analyses, the FS station antennas are pointed in random directions at elevation angles also randomly distributed between $+5^{\circ}$ and $-5^{\circ}$ as defined in [7]. The long term protection criterion applied to the UA FSS is $\mathrm{I} / \mathrm{N}=-$ $12 \mathrm{~dB}$ not to be exceeded for $20 \%$ of the time. The analyses performed to date show that the protection criterion is met for these bands.

The analysis of interference of UAES into FS station receivers is being performed for two uplink (earth-to-space) FSS bands (Table 1). In the 14.0$14.5 \mathrm{GHz}$ band there are primary allocations to the FS covering the entire band. 


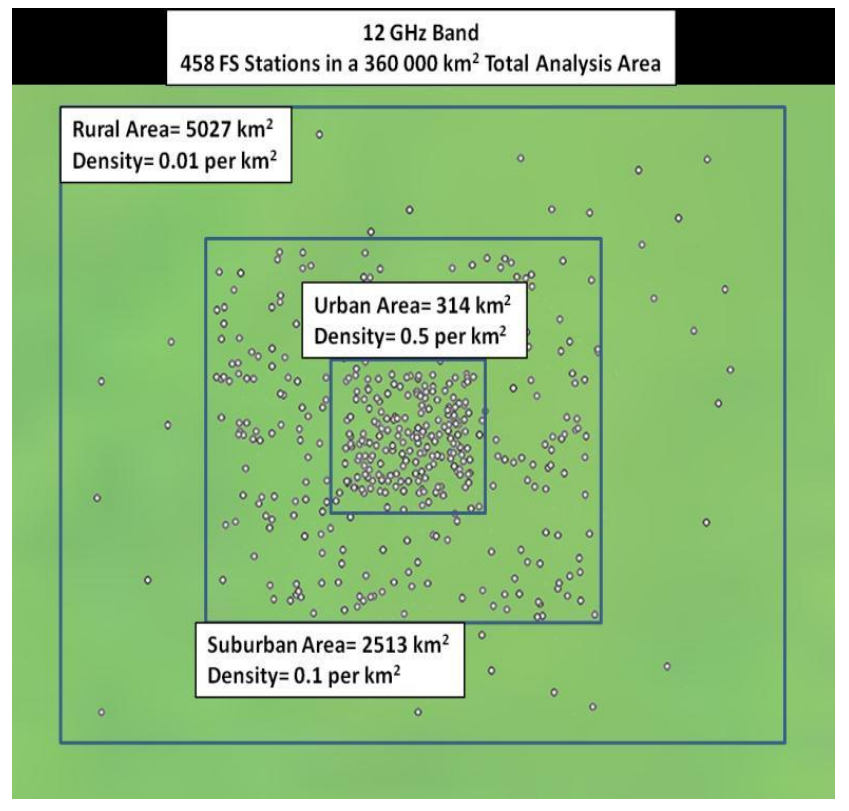

Figure 3. Example of a Distribution of FS Stations for an Assessment of Aggregate Interference into a UAES (from [8])

In the $27.5-30.0 \mathrm{GHz}$ band there are primary allocations to the FS covering 27.5-29.5 GHz. As with the space-to-earth bands, there are allocations to other terrestrial services in these bands but no technical characteristics for these other services have been documented, thus no sharing studies can be completed for these services.

The United States' analyses of interference in these two bands, being carried out by the National Aeronautics and Space Administration (NASA) spectrum, considers the interference effects from UAES transmitters in these bands. The FS transmission parameters used are again those defined in [7]. For these analyses, based on the expected densities of UA [1], it is possible for more than one UA to be within the line of sight of a FS station, hence the effects of several UA must be analyzed. In order to simulate the effects of various locations of UA relative to the FS station receiver, many UA locations are simulated in order to develop a cumulative distribution of interference level. Figure 4 shows an example of such a simulation. In this example, the FS station receive antenna is pointed due North, while UAES transmit antennas are pointing to a geostationary satellite located at the same longitude as the FS station, with the FS located at a latitude of $40^{\circ} \mathrm{N}$ and the UA at an altitude of $19,000 \mathrm{ft}$.

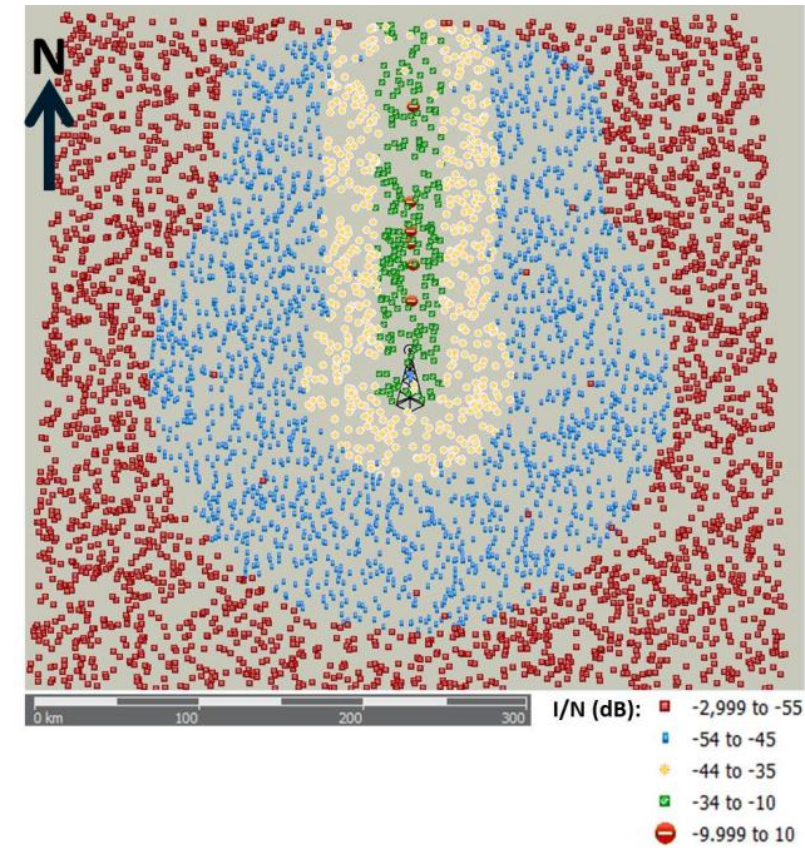

Figure 4. Example of UA locations for simulation of interference into FS station receiver

Visualyse Professional software [9] is being used to investigate this interference scenario for both frequency bands. The simulations are being performed at latitudes of $10^{\circ}, 40^{\circ}$, and $70^{\circ} \mathrm{N}$ with projected UAES densities. The long term protection criterion applied to the FS station is $\mathrm{I} / \mathrm{N}=-10 \mathrm{~dB}$ not to be exceeded for $20 \%$ of the time. Figure 5 shows an example of the cumulative distribution resulting from these analyses. Preliminary results are showing that for both frequency bands the protection criterion is expected to be met with a very substantial margin.

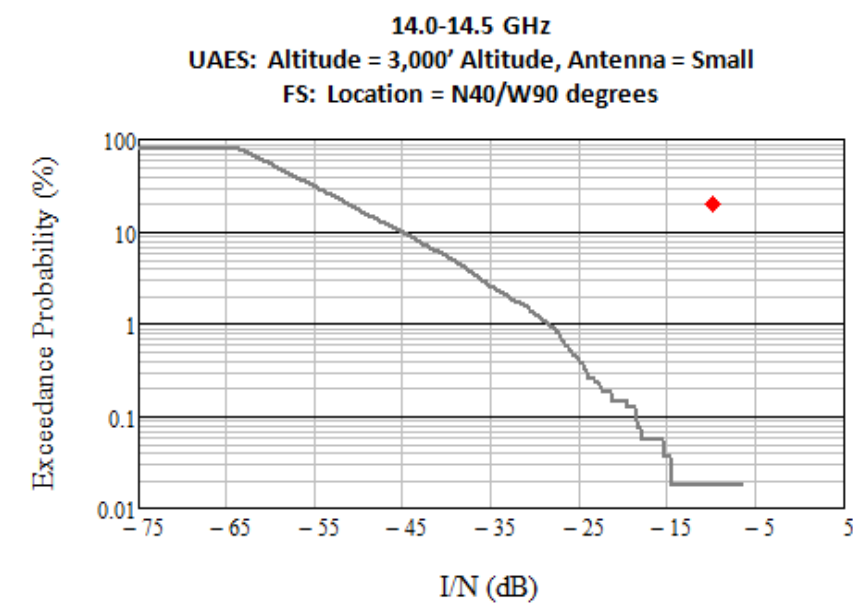

Figure 5. Example of cumulative distribution of I/N for UAES interference into FS station receiver 
At the current point in the preparation process for WRC-15, the sharing study analysis results are considered preliminary until agreed to by ITU-R delegates. Additional studies or changes to the analysis parameters may be required as a result of deliberation in ITU-R preparatory meetings.

\section{UAS CNPC Standards and Technology Development}

Activities are now underway to develop standards and technologies to enable the use of UAS CNPC spectrum. RTCA Special Committee 228 (SC-228) has recently been established to prepare Minimum Operational Performance Standards (MOPS) for unmanned aircraft systems. MOPS will be developed first for the terrestrial LOS CNPC system. When WRC-15 has clarified the situation for BLOS satellite CNPC, SC-228 will prepare MOPS for the satellite case. The MOPS will provide for certification of UAS using CNPC links for integration into non-segregated airspace.

ICAO is gathering information to begin the process of planning the use of the $5030-5091 \mathrm{GHz}$ band, as this band is designated for both LOS terrestrial and BLOS satellite use. SARPS for global interoperability of CNPC systems, both LOS terrestrial and BLOS satellite will follow. Activities carried out by RTCA, EUROCAE and other groups will contribute to this process. All of these activities are necessary to enable a path to certification of UAS inclusive of the CNPC link.

NASA's UAS in the NAS Project has been performing air-ground channel measurements in both of the terrestrial UAS CNPC bands in order to develop high fidelity channel models [10]. The channel models will enable accurate simulation of the performance of the terrestrial CNPC systems. To date, five of six planned air-ground measurement flight tests have been completed.

The UAS in the NAS Project has also developed and tested a first generation L-Band terrestrial CNPC prototype radio in order to provide technical performance characteristics to the standards development process. Seven flight tests of the first generation radio were performed during May and June, 2013 [11]. The test results indicated that the radios met or exceeded design specifications. Depending on aircraft altitude and ground terminal height, slant ranges of $100 \mathrm{nmi}$ to $130 \mathrm{nmi}$ were achieved, essentially to the edge of radio line of sight horizon, exceeding the $69 \mathrm{nmi}$ goal of the radio design. Communications between the aircraft and the ground station was demonstrated down to an altitude of $200 \mathrm{ft}$. A second generation prototype radio will have dual L-Band and C-Band functionality and will be tested in the spring of 2014 .

\section{Conclusion}

UAS CNPC links will require a high level of performance to enable certification of UAS. Reliability, availability, and integrity of CNPC links must meet safety-of-life standards, as CNPC link loss or performance degradation has the potential to result in catastrophic UAS failure. ICAO has deemed the CNPC function to be safety critical and has mandated that aviation safety spectrum must be used for this function.

Studies have established spectrum requirements for both LOS terrestrial and BLOS satellite spectrum for UAS CNPC. These studies led to approval of a new $\mathrm{AM}(\mathrm{R}) \mathrm{S}$ allocation in the 5030-5091 MHz band by WRC-12. As a result, the LOS spectrum requirement for UAS CNPC can now be met.

For the BLOS satellite component of CNPC, meeting the spectrum requirement is a more difficult challenge. Although AMS(R)S spectrum is currently allocated in the 5030-5091 MHz band, it is insufficient to meet the total bandwidth requirement, and also unavailable for use as no satellite currently provides service in this band. Therefore it has been proposed that satellites in the Fixed Satellite Service can provide the required spectrum for UAS CNPC. WRC-12 established Agenda Item 1.5 for WRC-15 to make a determination whether FSS can provide BLOS CNPC service. Studies are on-going to show whether regulatory and technical criteria for such use can be met.

Activities are now underway to develop standards and technologies for the LOS terrestrial component of UAS CNPC. The outcome of WRC-15 will be followed by similar efforts of the BLOS satellite component. The completion of these national and international standardization efforts will finalize the criteria for use of UAS CNPC spectrum and comprise a critical enabling element toward the goal of routine access for UAS. 


\section{References}

[1] ITU-R Report M.2171, Characteristics of unmanned aircraft systems and spectrum requirements to support their safe operation in nonsegregated airspace, December 2009, http://www.itu.int/pub/R-REP-M.2171-2009.

[2] ITU-R Report M.2205, Results of studies of the $\mathrm{AM}(\mathrm{R}) \mathrm{S}$ allocation in the band $960-1164 \mathrm{MHz}$ and of the $\operatorname{AMS}(\mathrm{R}) \mathrm{S}$ allocation in the band 5030-5091 MHz to support control and non-payload communication links for unmanned aircraft systems, November 2010, http://www.itu.int/pub/R-REP-M.2205-2010.

[3] ITU-R Report M.2233, Examples of technical characteristics for unmanned aircraft control and nonpayload communications links, November 2011, http://www.itu.int/pub/R-REP-M.2233-2011.

[4] ITU-R Report M.2237, Compatibility study to support the line-of-sight control and non-payload communications link(s) for unmanned aircraft systems proposed in the frequency band 5 030-5 091 MHz, November 2011, http://www.itu.int/pub/RREP-M.2237.

[5] ITU-R Final Acts - WRC-12, Geneva http://www.itu.int/pub/R-ACT-WRC.9-2012.

[6] ITU-R Document 5B/100-E, Reply statement to ITU-R Working Party 5B - use of satellite radio links for the control and non-payload communications of unmanned aircraft systems under WRC-15 Agenda Item $\quad 1.5, \quad$ October 24, 2012 , https://www.itu.int/md/R12-WP5B-C-0100/en.

[7] ITU-R Report F.758-5, System parameters and considerations in the development of criteria for sharing or compatibility between digital fixed wireless systems in the fixed service and systems in other services and other sources of interference, March 2012, http://www.itu.int/rec/R-REC-F.758-5201203-I.

[8] ITU-R Annex 41 to Document 5B/304-E, Technical and operational characteristics, interference and regulatory environments associated with the use of frequency bands allocated to the fixed-satellite service not subject to Appendices 30, $30 \mathrm{~A}$, and 30B for the control and non-payload communications of unmanned aircraft systems in non-segregated airspace, July 15, 2013, http://www.itu.int/md/R12-WP5B-C-0304/en.

[9] Transfinite Systems Limited, http://www. transfinite.com.

[10] Shalkhauser, Kurt A., David W. Matolak and Robert J. Kerczewski, September, 2013, NASA Lband and C-band air-ground channel measurement and modeling, Information Paper 05, ICAO Aeronautical Communication Panel, $29^{\text {th }}$ Meeting of Working Group F, Nairobi, Kenya, http://legacy.icao.int/anb/panels/acp/wgdoclist.cfm?

MeetingID $=296$

[11] Shalkhauser, Kurt A., James H. Griner and Robert J. Kerczewski, September, 2013, Flight tests of first generation prototype CNPC radio, Information Paper 06, ICAO Aeronautical Communications Panel, 29 ${ }^{\text {th }}$ Meeting of Working Group F, Nairobi, Kenya, http://legacy.icao.int/anb/panels/acp/wgdoclist.cfm? MeetingID=296

32nd Digital Avionics Systems Conference

October 6-10, 2013 